\section{Rezidive bei Rektumkarzinom und synchronen Lebermetastasen}

\begin{abstract}
Lassen sich aus den Rezidivmustern Rückschlüsse über die optimale Behandlung von Patienten mit Rektumkarzinom und synchronen Lebermetastasen ziehen? Dieser Frage ging ein New Yorker Team um Jean Butte nach.
\end{abstract}

D ie vollständige Entfernung von Lebermetastasen eines Rektumkarzinoms ist Standard bei Patienten mit resezierbarer Erkrankung. Sie verbessert das Überleben im Vergleich zur alleinigen Chemotherapie. Allerdings kommt es bei $70 \%$ der Patienten zu Rezidiven, am häufigsten in Leber und Lunge. Unklar ist aber, welches die optimale Behandlung ist: eine Resektion mit adjuvanter/neoadjuvanter systemischer Chemotherapie oder eine Resektion mit perioperativer Chemotherapie und Beckenbestrahlung. Daher untersuchten die Autoren nun bei 185 Patienten mit Rektumkarzinom, die in einer prospektiven Datenbank registriert waren, die Rezidivmuster und das Überleben nach der Resektion von Lebermetastasen. Alle Patienten hatten sich zwischen 1990 und 2008 einer vollständigen Resektion des Primärtumors im Rektum und der Lebermetastasen unter- zogen, die höchstens zwölf Monate nach der ersten Operation aufgetreten waren. $97 \%$ dieser Patienten hatten eine Chemotherapie erhalten und $49 \%$ eine Beckenbestrahlung vor $(71,4 \%)$ oder nach $(28,6 \%)$ der Rektumresektion.

Das krankheitsspezifische 5-JahresÜberleben betrug in der gesamten Kohorte $51 \%$. Die mediane Beobachtungsdauer betrug 38 Monate. 130 Patienten (70\%) entwickelten innerhalb eines Jahres ein Rezidiv, das nur bei 18 Patienten $(10 \%)$ im Becken auftrat; elf davon hatten zusätzlich an anderen Stellen Rezidive. Die Rezidivmuster korrelierten nicht mit dem krankheitsspezifischen Überleben.

Außer dem Alter gab es weder klinische noch pathologische Unterschiede zwischen Patienten mit und ohne Rezidive im Becken. Auch eine Beckenbestrahlung war nicht damit assoziiert. Unabhängig von der Bestrahlung war

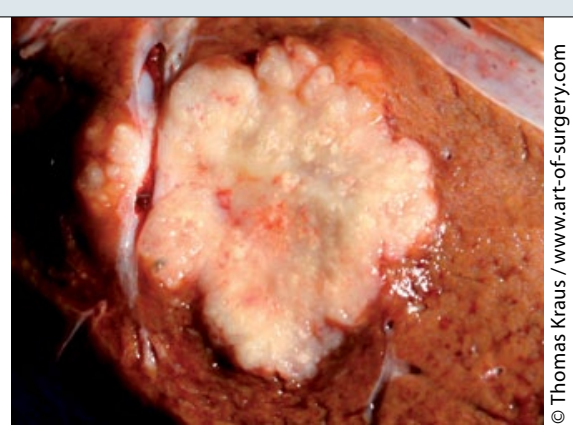

Die optimale Behandlung synchroner Lebermetastasen eines Rektumkarzinoms ist unklar.

das Risiko für ein Rezidiv im Becken signifikant niedriger als an anderen Stellen. Insgesamt waren die Rezidivraten aber bei Patienten mit und ohne Beckenbestrahlung vergleichbar.

Fazit: Bei Patienten mit reseziertem Rektumkarzinom und synchronen Lebermetastasen traten Rezidive wesentlich häufiger an anderen Stellen als im Becken auf. Das Risiko für Lokalrezidive war nicht mit einer Beckenbestrahlung assoziiert. Daher könnte ein Verzicht auf die Radiotherapie bei Patienten mit synchroner Erkrankung in Betracht gezogen werden.

Judith Neumaier

Butte JM et al. Patterns of failure in patients with early onset (synchronous) resectable liver metastases from rectal cancer. Cancer. 2012;118(21):5414-23.

\section{Prognose bei metastasiertem Darmkrebs verbessern}

Für die Therapie des metastasierten kolorektalen Karzinoms (mCRC) stehen viele verschiedene Optionen zur Verfügung. Prädiktive und prognostische Marker könnten die Therapieentscheidung unterstützen, so zum Beispiel zirkulierende Tumorzellen (CTC) und das karzinoembryonales Antigen (CEA).

$\mathrm{N}$ achdem die Autoren in einer Untersuchung bereits prospektiv Hinweise darauf fanden, dass CTC für die Prognose eines mCRC von Bedeutung sind, nahmen sie jetzt in einer zweiten Analyse die Bedeutung des Verhältnisses von CTC-Zahl und CEA für das Gesamtüberleben (OS) unter die Lupe. Ausgewertet wurden die Daten von 217 Patienten mit mCRC und einem CEA-Spiegel vor Therapie von $\geq 10 \mathrm{ng} / \mathrm{ml}$ sowie CEAMessungen zeitnah nach den jeweiligen CTC-Bestimmungen, die vor Therapiebeginn und danach immer wieder bis zu 20 Wochen erfolgt waren.
Ein erhöhter CEA-Spiegel ( $\geq 25 \mathrm{ng}$ / $\mathrm{ml})$ vor Therapiebeginn war mit einem kürzeren OS assoziiert (16,9 vs. 18,5 Monate bei $>25 \mathrm{ng} / \mathrm{ml}$; $\mathrm{p}=0,3408)$. Bei einem Grenzwert des CEA-Spiegels von 50 $\mathrm{ng} / \mathrm{ml}$ wurde diese Assoziation auch signifikant $(15,8$ Monate bei $\geq 50 \mathrm{ng} / \mathrm{ml}$ vs. 20,7 Monate bei < $50 \mathrm{ng} / \mathrm{ml}$; $\mathrm{p}=0,012$ ). Bei den 157 Patienten (75\%) mit einem Ausgangs-CEA-Wert von $\geq 25 \mathrm{ng} / \mathrm{ml}$ fand sich bei einer geringen Zahl von CTC $(<3, \mathrm{n}=99)$ mit im Median 20,8 Monaten ein signifikant längeres Überleben als bei denjenigen mit mehr CTC $(\mathrm{n}=58 ; 11,7$ Monate, $\mathrm{p}=0,001)$. Im Ver- lauf - gemessen nach 3-5 bzw. 6-12 Wochen - war die Zahl der CTC ( $<3$ bzw. $\geq$ 3) unabhängig vom CEA-Wert prognostisch relevant. Nach der multivariaten Analyse war von den Ausgangswerten nur die Zahl der CTC, nicht aber der CEA-Wert, ein Prädiktor für das Überleben. 6-12 Wochen nach Therapiebeginn waren CEA und CTC unabhängig voneinander prognostisch für das OS bedeutsam.

Fazit: CTC und CEA können die prognostische Einschätzung beim mCRC unterstützen. $\mathrm{Ob}$ dies für therapeutische Entscheidungen bedeutsam ist, müssen weitere Studien zeigen. Wichtig ist nach Ansicht der Autoren jetzt, diese Marker in prospektive Therapiestudien mit einzubeziehen.

Friederike Klein

Aggarwal $C$ et al. Relationship among circulating tumor cells, CEA and overall survival in patients with metastaticcolorectal cancer. Ann Oncol. 2013;24(2):420-8. 